PISA IFUP-TH XX, June 1993

\title{
The Kramers equation simulation algorithm: II. An application to the Gross-Neveu model
}

\author{
Matteo Beccaria ${ }^{(1,2)}$, Giuseppe Curci ${ }^{(2,1)}$, Luca Galli(1) \\ (1) Dipartimento di Fisica, Universitá di Pisa \\ Piazza Torricelli 2, I-56100 Pisa, Italy \\ (2) I.N.F.N., sez. di Pisa \\ Via Livornese 582/a, I-56010 S. Piero a Grado (Pisa) Italy
}

(October 14, 2018)

\begin{abstract}
We continue the investigation on the applications of the Kramers equation to the numerical simulation of field theoretic models. In a previous paper we have described the theory and proposed various algorithms. Here, we compare the simplest of them with the Hybrid Monte Carlo algorithm studying the two-dimensional lattice Gross-Neveu model. We used a Symanzik improved action with dynamical Wilson fermions. Both the algorithms allow for the determination of the critical mass. Their performances in the definite phase simulations are comparable with the Hybrid Monte Carlo. For the two methods, the numerical values of the measured quantities agree within the errors and are compatible with the theoretical predictions; moreover, the Kramers algorithm is safer from the point of view of the numerical precision.
\end{abstract}

PACS numbers: 11.15.Ha, 05.50.+q, 11.30.Na, 11.30.Rd 


\section{INTRODUCTION}

In a previous work [1], we used a compact operatorial formalism to study the applicability of the Kramers equation to the numerical simulation of quantum field theories on the lattice. In the standard Monte Carlo approach, we must generate ensembles of states distributed according to a given statistical weight. In [1], our strategy has been to realize a diffusive process involving the physical degrees of freedom but also auxiliary variables which drive the diffusion. The final equilibrium distribution approximates the desired one. The resulting schemes can be made exact by a global accept-reject test and provide thus generalizations of the usual Hybrid Monte Carlo [2,3] and of the exact hyperbolic algorithm [5]. For these algorithms, a good behaviour with increasing volume and from the point of view of numerical precision is expected. Moreover, in [1] we have shown how the freedom in the auxiliary variables sector may be used to reduce significantly the autocorrelations in the free field case. In this paper we study the behaviour of the simplest among the proposed schemes. We take the Hybrid Monte Carlo as a reference point. Our theoretical laboratory is the two-dimensional $N$-fermion Gross-Neveu model with Wilson fermions [6]. This model is an asymptotically free purely fermionic model with a discrete $\gamma_{5}$ symmetry whose dynamical breakdown leads to mass generation. It has an exact large $N$ solution and is $1 / N$ expandable [7] allowing for analytical investigation of its non perturbative properties.

In our lattice simulation of the model we use the Wilson formulation to avoid the doubling problem and let the fermion number $N$ be free. However, using Wilson fermions, a bare mass must be introduced and tuned in order to restore the chiral symmetry in the continuum limit. The restoration of chiral symmetry is a significant test for new algorithms: previous works [6,8] showed that non exact algorithms (Langevin, pseudofermions) fail to locate the critical point by means of mixed-phase techniques. In this work we shall show that the algorithm based on the Kramers equation does not encounter this problem. The use of two-dimensional four-fermions models as prototypes for realistic theories like $Q C D$ is somewhat limited. However, a non trivial model with a discrete chiral symmetry breaking 
and many dynamical fermionic degrees of freedom implies enough problems to be a severe test for our algorithms.

In Sec. [I] we introduce the Gross-Neveu model. In Sec. [II] we describe explicitly the algorithms that we have used. In Sec. IV we discuss how we compared them. In Sec. D we review the previous simulation of the Kramers algorithm and of the Symanzik improved Gross-Neveu model. We then describe our explicit numerical simulation and present the results. Finally, Sec. V1 is devoted to a short summary and conclusions.

\section{THE GROSS-NEVEU MODEL}

The action of the model in the continuum is

$$
S=\int d^{2} x\left(\bar{\psi}^{(\alpha)} \not \partial \psi^{(\alpha)}-\frac{1}{2} g^{2}\left(\bar{\psi}^{(\alpha)} \psi^{(\alpha)}\right)^{2}+m \bar{\psi}^{(\alpha)} \psi^{(\alpha)}\right) \quad \alpha=1 \cdots N
$$

where $\psi^{(\alpha)}$ is a multiplet of two-dimensional Dirac fermions.

Rewriting the quartic interaction with a Lagrange multiplier we obtain

$$
S=\int d^{2} x\left(\bar{\psi}^{(\alpha)} \not \partial \psi^{(\alpha)}+(\sigma+m) \bar{\psi}^{(\alpha)} \psi^{(\alpha)}+\frac{1}{2 g^{2}} \sigma^{2}\right)
$$

The manifest $U(N)$ symmetry of the model can be enlarged to $O(2 N)$ by writing the fermionic fields in terms of their hermitean Majorana components. We refer to 110 for a discussion of the renormalization properties of the continuum model in the two formulations with and without the auxiliary $\sigma$ field. In the chiral limit the model enjoys the discrete symmetry

$$
\psi \rightarrow \gamma_{5} \psi \quad \bar{\psi} \rightarrow-\bar{\psi} \gamma_{5} \quad \sigma \rightarrow-\sigma
$$

As a consequence, the potential of $\sigma$, related to that of the composite field $\bar{\psi} \psi$, is symmetric under the exchange $\sigma \rightarrow-\sigma$ and possesses two degenerate minima. The chiral symmetry is spontaneously broken. The same holds on the lattice, but in the Wilson formulation we need an explicit mass term to control mass renormalization. 
The $1 / N$ expansion of the model is obtained by integrating out the fermion fields. By introducing the large- $N$ coupling $\lambda=N g^{2}$ and the field $\Sigma=\sigma+m$, the corresponding "effective" action is

$$
S_{e f f}=N \int d^{2} x\left(\frac{1}{2 \lambda}(\Sigma-m)^{2}-\operatorname{Tr} \log M(\Sigma)\right) \quad M(\Sigma)=\not \partial+\Sigma
$$

In a perturbative expansion in powers of $g$, the fermions remain massless in the chiral model. However, already in the leading order of the $1 / N$ expansion, a non-zero expectation value of the $\Sigma$ field is dynamically generated playing the role of a non-perturbative fermion mass. From the study of the phase diagram of the model we can infer the existence of a critical point in the weak coupling region. At this point the chiral symmetry can be recovered (and dynamically broken) with an appropriate choice of the bare critical mass. The vacuum expectation value $\langle\Sigma\rangle$ is exponentially depressed as $\lambda \rightarrow 0$ following the leading renormalization group prediction $\langle\Sigma\rangle= \pm \Lambda \exp (-\pi / \lambda)$. Actually, on a finite lattice at non zero $\lambda$ one chooses the perturbative mass in order to have a double well shaped potential for the $\Sigma$ field having two degenerate minima. The $\Sigma \rightarrow-\Sigma$ symmetry is recovered only in the chiral limit; this is reminiscent of the doubling problem since one has exactly

$$
\langle F(\psi, \bar{\psi}, \Sigma, r)\rangle=\left\langle F\left(\gamma_{5} \psi,-\bar{\psi} \gamma_{5},-\Sigma,-r\right)\right\rangle
$$

where $r$ is the Wilson (or Symanzik) coefficient of the mass term which solves the doubling. Quantities like the asimmetry in the steepness of the potential in the two minima can be $1 / N$ expanded and computed.

Since we are in two dimensions, finite volume effects are particularly dangerous. Therefore we improved the lattice Wilson action at the tree-level following the method of Symanzik [1]. The resulting action improved at order $O\left(a^{2}\right)$ is

$$
\begin{aligned}
S= & \sum_{n \mu} \bar{\psi}_{n}\left[\gamma_{\mu}\left(\frac{2}{3}\left(\psi_{n+\hat{\mu}}-\psi_{n-\hat{\mu}}\right)-\frac{1}{12}\left(\psi_{n+2 \hat{\mu}}-\psi_{n-2 \hat{\mu}}\right)\right)+\right. \\
& \left.+\frac{r_{s}}{2}\left(\psi_{n+2 \hat{\mu}}-4 \psi_{n+\hat{\mu}}+6 \psi_{n}-4 \psi_{n-\hat{\mu}}+\psi_{n-2 \hat{\mu}}\right)\right]+ \\
& +\sum_{n}\left[\bar{\psi}_{n} \psi_{n}\left(m+\sigma_{n}\right)+\frac{N}{2 \lambda} \sigma_{n}^{2}\right]
\end{aligned}
$$


where we have understood the flavour indices. The Fermi fields have antisymmetric boundary conditions in the temporal direction. The parameter $r_{s}$ is the Wilson parameter for the Symanzik action. We have taken $r_{s}=1 / 3$ remarking that the strong coupling matching between Wilson and Symanzik actions is at $r_{s}=1 / 3 r_{w}$. In [6] the interested reader can find a detailed account of the leading and next-to-leading $1 / N$ expansion of the model described by Eq. (2.6). In momentum representation, the fermionic matrix is

$$
M=i \gamma_{\mu} \bar{p}_{\mu}+M_{S}(p)+\Sigma
$$

where we have defined

$$
\begin{aligned}
M_{S}(p) & =\frac{1}{2} r_{s} \sum_{\mu} \hat{p}_{\mu}^{4}, \\
\hat{p}_{\mu} & =2 \sin \frac{p_{\mu}}{2} \\
\bar{p}_{\mu} & =\sin p_{\mu}\left(1+\frac{1}{6} \sum_{\mu} \hat{p}_{\mu}^{2}\right),
\end{aligned}
$$

On a finite $L \times T$ lattice, the form Eq. (2.4) of the effective action together with Eq. (2.7) allows for the computation of any relevant quantity in the leading $1 / N$ limit.

\section{DESCRIPTION OF THE ALGORITHMS}

We now describe in an unified scheme the algorithms that we have used. We give all the details showing how the fermionic fields are dealed with. Because of the particular structure of $M(\Sigma)$, we may introduce $N$ real auxiliary fields $\chi^{(\alpha)}(\alpha=1 \ldots N$ and Dirac indices understood) and conjugate bosonic momenta $\pi$ to form the extended action

$$
S=\sum_{\text {sites }}\left(N \frac{(\Sigma-m)^{2}}{2 \lambda}+\frac{1}{2} \pi^{2}+\frac{1}{2} \chi^{(\alpha)}\left[M(\Sigma)^{T} M(\Sigma)\right]^{-1} \chi^{(\alpha)}\right)
$$

For each given configuration of $\Sigma$ and of the auxiliary pseudofermionic fields $\chi$, we define the following $N$ fields

$$
\Phi^{(\alpha)}(\Sigma)=\left[M(\Sigma)^{T} M(\Sigma)\right]^{-1} \chi^{(\alpha)},
$$


where the sparse matrix inversion is performed by means of the Conjugate Gradient algorithm

The $\chi$ fields action is quadratic. Therefore, we can extract randomly the $\chi$ fields according to their exact equilibrium distribution and evolve the other fields between two successive such refreshments. Note that this procedure introduces another free parameter, namely the frequency of the $\chi$ fields update.

The single sweep updating is given by the following algorithm [1]

- Refresh the auxiliary $\chi^{(\alpha)}$ according to

$$
\left\{\begin{array}{l}
\chi^{(\alpha)}=M^{T}(\Sigma) \eta^{(\alpha)} \\
\pi=\eta
\end{array}\right.
$$

Here, $\eta^{(\alpha)}$ and $\eta$ are vectors of gaussian random numbers with zero mean and unit variance. This step must be done only every $k$ sweeps.

- Update the momenta $\pi$ according to

$$
\pi^{\prime}=e^{-\gamma \epsilon} \pi+\sqrt{1-e^{-2 \gamma \epsilon}} \xi
$$

where $\xi$ is a gaussian random number with zero mean and unit variance. The parameters $\gamma, \epsilon$ are positive real numbers.

- Integrate the equations of motion associated with the force

$$
F_{n}(\Sigma)=-\frac{N}{\lambda}\left(\Sigma_{n}-m\right)+\frac{1}{2} \Phi^{(\alpha)} \frac{\delta}{\delta \Sigma_{n}}\left(M^{T}(\Sigma) M(\Sigma)\right) \Phi^{(\alpha)}
$$

( $n$ is a site index) using $N_{m d}$ iterations of the leap-frog scheme

$$
\begin{aligned}
\pi(t+\epsilon / 2) & =\pi(t)+\varepsilon / 2 F(\Sigma(t)), \\
\Sigma(t+\epsilon) & =\Sigma(t)+\varepsilon \pi(t+\epsilon / 2), \\
\pi(t+\epsilon) & =\pi(t+\epsilon / 2)+\varepsilon / 2 F(\Sigma(t+\epsilon)),
\end{aligned}
$$

or its dual form in which the roles of $\Sigma$ and $\pi$ are interchanged. 
- Perform a Metropolis test between the states before and after the integration of the equations of motion. If the test fails, reject the proposal for the $\Sigma$ field and negate all the momenta

$$
\Sigma \rightarrow \Sigma_{\text {old }} \quad \pi \rightarrow-\pi_{\text {old }}
$$

otherwise accept the proposal for both $\Sigma$ and $\pi$.

We have four free parameters: $k, N_{m d}, \epsilon$ and $\gamma$. Detailed Balance is exactly satisfied for each set of values they take. The algorithm under study (which we shall call "Kramers algorithm") is obtained with $N_{m d}=1$ and arbitrary values of $k, \gamma$ and $\epsilon$. The usual Hybrid Monte Carlo corresponds to $k=1, \gamma \rightarrow \infty$ and arbitrary values of $N_{m d}$, $\epsilon$. Of course, in the Hybrid Monte Carlo case, there is no mixing between the old and new momenta and it is unnecessary to negate them on rejection.

We remark that from a physical point of view, the Kramers algorithm possesses two distinct time steps, $\epsilon_{i r r}=\epsilon \gamma$ drives the "irreversible" motion, whereas $\epsilon_{r e v}=\epsilon$ drives the "reversible" one. Apart from effects coming from the negation of momenta, we expect that the Kramers and the Hybrid Monte Carlo algorithms to behave similarly when $\epsilon_{i r r} \sim 1 / N_{m d}$ with $\epsilon_{\text {rev }}=\epsilon_{m d}$.

\section{COMPARISON}

In [1] we argued that the Kramers algorithm should behave better than the Hybrid Monte Carlo for large volumes. The argument is rather naive and must be confirmed numerically. Consider a lattice model satisfying $L \gg \xi$, where $L$ is the lattice size and $\xi$ the correlation length. On general grounds, the acceptance rate of the Hybrid Monte Carlo is known to be [3]

$$
P_{a c c} \sim \operatorname{erfc}\left(c N_{m d} \epsilon^{3} \sqrt{V}\right) .
$$

Optimal tuning is expected to require $N_{m d} \sim 1 / \epsilon$ giving a sweep-sweep correlation $\tau \sim 1 / \epsilon$. If the volume is varied, we have to scale $\epsilon \sim V^{-1 / 4}$ in order to keep the acceptance probability 
constant. The advantage of the Kramers algorithm is that $N_{m d}=1$. If we neglect the influence of the momenta negation on the acceptance, then the scaling relation is modified to $\epsilon \sim V^{-1 / 6}$. If, moreover, the optimal autocorrelations stay $\sim 1 / \epsilon$ then it follows that on large volumes the Hybrid Monte Carlo behaves worst than Kramers.

Empirically the maximum acceptable value for $\epsilon$ in the Kramers algorithm was rather greater than the one of the Hybrid Monte Carlo. This is reasonable because, at fixed $\epsilon$, as $N_{m d}$ is increased the acceptance rate decreases before reaching a plateaux.

Another advantage of the Kramers algorithm is related to the numerical precision, the opportunity of having $N_{m d}=1$ without penalty is surely welcome; for instance, in $Q C D$, as the volume $\left(L^{4}\right)$ grows or the quark mass $(m)$ is decreased we must have $N_{m d} \sim L / m^{3 / 4}$ and some protection seems necessary to protect irreversibility against the accumulation of numerical errors.

To compare quantitatively the performances of the two algorithms we must determine which is the computer time needed to have a given statistical error. If we denote with $\tau_{\Omega}$ the integrated autocorrelation of the observable $\Omega$, then the computational cost of an algorithm measuring $\Omega$ is proportional to $2 \tau_{\Omega}$ which is the reduction factor which determines the actual number of independent measures. This factor must be multiplied by the number of Conjugate Gradient inversions needed to make a single sweep. Indeed, in a model with dynamical fermions it is reasonable to neglect the time expended during all the other steps of the algorithm. In our scheme we have blocks each made of $N_{m d}$ molecular dynamics steps followed by a Metropolis test and we refresh the pseudofermionic fields $\chi$ only at the end of $k$ such blocks. Let us call $L F_{1}$ and $L F_{2}$ the two dual second order leap-frog schemes (see [1] for a compact review of symplectic integrators) which we sketch as follows

$$
\begin{aligned}
& L F_{1}: \pi\left(t_{0}\right) \rightarrow \Sigma(t+\epsilon / 2) \rightarrow \pi(t+\epsilon), \\
& L F_{2}: \Sigma\left(t_{0}\right) \rightarrow \pi(t+\epsilon / 2) \rightarrow \Sigma(t+\epsilon) .
\end{aligned}
$$

Keeping $\chi$ fixed, a new Conjugate Gradient inversion is needed only when the $\Sigma$ field configuration or the $\chi$ fields change. The advantage of the $L F_{1}$ scheme is that it computes the 
old and new action at the same evolution times at which the force itself must be evaluated. The quantity determining the computational cost is the number of inversions per sweep computed between two refreshment of $\chi$

$$
\begin{aligned}
& \mathcal{C}\left(k, N_{m d}\right)_{L F_{1}}=N_{m d}+1 / k, \\
& \mathcal{C}\left(k, N_{m d}\right)_{L F_{2}}=N_{m d}+1+1 / k,
\end{aligned}
$$

and the actual performance is just this ( $\Omega$ independent) factor corrected by the ( $\Omega$ dependent) autocorrelation. From Eq.(4.3) we see that $L F_{2}$ must certainly be ruled out at small $N_{m d}$. For what concerns $k$, in the Hybrid Monte Carlo case the $1 / k$ term is usually not very important because $N_{m d} \gg 1$. However, with the Kramers algorithm, a large $k$ is twice better than $k=1$. Moreover $k>1$ may allow for a more efficient exploration of the phase space.

We remark that our conclusions hold provided that $L F_{1}$ and $L F_{2}$ have the same behaviour concerning autocorrelations.

\section{SIMULATION AND NUMERICAL RESULTS}

\section{A. Review of previous results}

\section{Previous study of the Kramers algorithm}

In [5], the Kramers algorithm was applied to the simulation of quenched compact $Q E D$ on a $8^{4}$ lattice in the disordered phase. Measures of the average plaquette and of the average $2 \times 2$ Wilson loop were made using also the Hybrid Monte Carlo algorithm as a reference method. The performances of the two algorithms turned out to be comparable.

\section{Previous study of the improved Gross-Neveu model}

In [6], the Symanzik improved Gross-Neveu model was studied on the lattice using the pseudofermion algorithm. In this scheme the variation of the fermionic contribution to the 
action is expanded to the lowest order in the $\Sigma$ field variation $\delta \Sigma$.

$$
\delta S \rightarrow \frac{N}{2 \lambda}(\Sigma-m)^{2} \delta \Sigma+\frac{N}{2 \lambda} \delta \Sigma^{2}-N \operatorname{Tr} M^{-1}(\Sigma) \delta \Sigma .
$$

The fermionic matrix inversion was performed computing

$$
M_{i j}^{-1}=\left\langle\chi_{i} \chi_{j}^{\dagger}\right\rangle
$$

as a Monte Carlo average in presence of the quadratic action $S_{\chi}=\chi^{\dagger} M^{-1} \chi$. The systematic error to be kept under control has two contributions. It gets an easily controllable statistical contribution from the inversion and a difficult contribution due to the truncation of the fermionic term at small but non zero $\delta \Sigma$. An extrapolation as $\left\langle(\delta \Sigma)^{2}\right\rangle \rightarrow 0$ must be performed. Using the mixed-phase tecnique, the critical bare mass $m_{c}$ was determined at different values of the parameters and of the step $\left\langle(\delta \Sigma)^{2}\right\rangle$. The extrapolation did not agree with the theoretical prediction in the case of $\langle\Sigma\rangle$ and was explained as a wrong determination of $m_{c}$. Moreover, deviations from the Schwinger-Dyson equations of the model did not show the expected linear dependence on $\left\langle(\delta \Sigma)^{2}\right\rangle$. These problems were absent for $\left\langle\Sigma^{2}\right\rangle$ which is expected to have a milder dependence on $m_{c}$.

In [8], the same kind of measurements were done using two different algorithms: the Langevin one with bilinear noise and the Hybrid Monte Carlo. In both cases, the inversion of the fermionic matrix was performed by the Conjugate Gradient algorithm. The Langevin algorithm is not exact, given a step $\epsilon$ the update of the bosonic field is computed as

$$
\begin{aligned}
\Sigma(x, t+\epsilon)= & \Sigma(x, t)-\epsilon \frac{\delta S}{\delta \Sigma(x, t)}+\sqrt{\epsilon} \eta(x, t)+ \\
& +\epsilon \sum_{y, z, w} \eta_{f}(y, t) M_{y z}^{-1} \frac{\delta M_{z w}}{\delta \Sigma(x, t)} \eta_{f}(w, t),
\end{aligned}
$$

where $\eta$ and $\eta_{f}$ are gaussian fields normalized as

$$
\left\langle\eta(x, t) \eta\left(x^{\prime}, t^{\prime}\right)\right\rangle=2 \delta_{x x^{\prime}} \delta_{t t^{\prime}}
$$

An extrapolation as $\epsilon \rightarrow 0$ is needed. The Langevin algorithm was found to be completely unable to determine the critical mass since the critical point was so unstable that different 
seeds of the random number generator gave different results. Using the theoretical value for the critical mass the simulation in the definite phase was stable only for the smallest values of $\epsilon$. On the other hand, the Hybrid Monte Carlo simulations turned out to be perfectly stable in both the mixed and definite phase simulations. The results (included the stable Langevin runs) were consistent with the theoretical predictions.

\section{B. Our results}

In this work, we have repeated the measures of [6,8] using our favoured algorithm. Moreover we have studied the variation of the performance as the tuning is varied, an information which is very important from a practical point of view.

The numerical simulation has been done on the APE supercomputer [15]. The model operating at Pisa is the so called "tube" machine, a 128 processor parallel computer with a peak performance of 6 GigaFlops. All the code has been written in the high-level "apese" language [16].

We have used the Conjugate Gradient algorithm (see [13,14 for a discussion oriented to the Dirac lattice operator) to obtain iteratively the inverse of the sparse symmetric matrix $A=M^{T}(\sigma) M(\sigma)$. We did not use any kind of preconditioning, the improved Symanzik action has next-to-neighbours interactions and an efficient incomplete factorization is not trivial. Moreover, the condition number of $A$ becomes larger when $\Sigma \rightarrow 0$, but at $\lambda=2.0$

on the $40^{2}$ lattice, the average $\Sigma$ (which is the fermion mass) and its fluctuations provide a good rate of convergence and the inversion problem was not critical. We tried also to invert directly the matrix $M$ using the Biconjugate Gradient algorithm [12] but we did not see any advantage. The stopping condition in the Conjugate Gradient algorithm is usually chosen to be a bound like

$$
\|r\| \leq \varepsilon_{C G}\|b\|
$$

$r$ is the residual vector: in the following we shall denote with $r_{n}$ the recursive residual computed on fly by the algorithm and we shall denote with $\tilde{r}_{n}$ the residual defined by 


$$
\tilde{r}_{n}=b-A x_{n} .
$$

We stress an important fact: let $x^{*}$ be the exact solution, the Conjugate Gradient algorithm guarantees that $\left\|x_{n}-x^{*}\right\|$ monotonically decreases. On the other hand $\left\|r_{n}\right\|$ and $\left\|\tilde{r}_{n}\right\|$ may show relatively large fluctuations. Hence a decreasing trend must be checked on the succession of residuals to keep under control this effect. Let us now address the problem of numerical precision. In absence of rounding errors there would be no difference at all between the two residuals $r_{n}$ and $\tilde{r}_{n}$ as can be checked inductively using the explicit form of the algorithm. The effect of rounding errors associated to a finite precision machine can be easily studied on simple model problems (like harmonic problems on regular lattices). The result is that, given the exact solution $x^{*}$, the recursive residual $r_{n}$ tends to zero, while the true error has a positive infimum

$$
\frac{\left\|x_{n}-x^{*}\right\|}{\left\|x^{*}\right\|} \rightarrow \varepsilon \quad \text { as } \quad n \rightarrow \infty
$$

of the order of the machine precision. The small number $\varepsilon$ may be further reduced if all the scalar products in the algorithm are implemented as binary sums. The same saturation phenomena occurs to the residual $\tilde{r}$, namely

$$
\left\|\tilde{r}_{n}\right\| \rightarrow \varepsilon^{\prime}\|b\| \quad \text { as } n \rightarrow \infty .
$$

Here $\varepsilon^{\prime}$ is a number which depends on the machine word length, but also on the norm of $A$. Typically the saturation of $\| x_{n}-x^{*}|| /|| x^{*}||$ and $\left\|\tilde{r}_{n}\right\|$ happens roughly at the same time. In a realistic problem $x^{*}$ is not known and one can study the behaviour of $r_{n}$ and $\tilde{r}_{n}$. The departure of $r_{n}$ from $\tilde{r}_{n}$ is typically very slow until the machine precision is reached and saturation sets up. Our stopping condition has been

$$
\left\|r_{n}\right\|<10^{-8}
$$

and we checked on configurations taken randomly during the runs that the saturation of $\left\|\tilde{r}_{n}\right\|$ was reached. 
We used a $40^{2}$ lattice with $N=10$ flavours. In the framework of the $1 / N$ expansion one can study the width of the scaling window, which is roughly measured by looking at the value of $\Sigma$ in the crossover region where the weak and strong coupling expressions for the perturbative mass are equal. A detailed discussion of the scaling properties of the model including next-to-leading $1 / N$ corrections and finite size effects can be found in [6]. We have chosen $\lambda=2.0$ at which the correlation length is about three lattice spacings and we are in the scaling window. The volume factor in favor of the improved Symanzik action is about 15 and at $L=40$ we expect from the finite lattice $1 / N$ prediction to have very small finite size effects.

We determined the critical Wilson mass at which a first order transition takes place using mixed-phase runs and measured in the two definite phases $\langle\Sigma\rangle$ and the composite $\left\langle\Sigma^{2}\right\rangle_{c}$ which provides informations on the steepness of the vacua. For these quantities the next-to-leading $1 / N$ corrections [6] can be computed and compared with the numerical simulation.

On the $40^{2}$ lattice the theoretical predictions are $m_{c}=-0.974$ and

$$
\begin{array}{ll}
\left\langle\Sigma_{-}\right\rangle=-0.293, & \left\langle\Sigma_{-}^{2}\right\rangle_{c}=0.190, \\
\left\langle\Sigma_{+}\right\rangle=0.311, & \left\langle\Sigma_{+}^{2}\right\rangle_{c}=0.146 .
\end{array}
$$

We started taking $k=1$. After a very rough tuning of $\gamma$ and $\epsilon$ using definite phase runs with the $1 / N$ theoretical mass, we choose $\gamma=5.0$ and $\epsilon=0.06$ for the mixed-phase runs. Setting half of the lattice to the positive vacuum and the other half to the negative one, after a short thermalization, a kink configuration appears which is very long-lived when the bare mass is at its critical value. From inspection of the leading effective potential one checks that bare masses smaller than the critical one favours the negative vacuum and viceversa. In Fig.(I) we show the time evolution of $\langle\Sigma\rangle$ for 16 different values of the bare mass starting from $m_{c}=-0.958$ separated by $\Delta m_{c}=0.002$. The result is

$$
m_{c}=-0.969 \pm 0.002,
$$

In this part of the simulation we did not insist on a heavy tuning of $\gamma, \epsilon$ and $k$ because we were mainly interested in the exact determination of $m_{c}$. We then turned to a detailed 
tuning of $\gamma$ and $\epsilon$ using definite phase runs at the critical point. During these runs $k$ was set to the unoptimized value 6 . In Tab. (四) we report measures of autocorrelations obtained in the two phases of the model with different values of $\gamma$ and the common choice $\epsilon=0.09$. $P_{a c c}$ was about $70-75 \%$ and greater values $\epsilon$ did show a sharp decrease in $P_{a c c}$. We did not find any sistematic dependence of $\gamma$ for the two measured expectation values of $\langle\Sigma\rangle$ and $\left\langle\Sigma^{2}\right\rangle_{c}$.

All autocorrelations are computed with the automatic windowing algorithm [17] by varying the $c$ constant in the range 4.0-8.0, cross-checking the result with the statistical inefficiency [18] and using consistently at least $1500 \tau$ sweeps.

In the mixed-phase, unoptimized Hybrid Monte Carlo runs gave the same value for the critical mass. We did not compare the performances because optimization in mixed-phase runs was too time consuming. In the definite phase runs we have taken $\epsilon=0.06$ and $N_{m d}=8$ corresponding to a ratio of $\mathcal{C}$ factors between the two algorithms of about 7.7 . The related rescaled autocorrelations are shown in Tab. (III). The resulting expectation values were always consistent with the Kramers algorithm and the $1 / N$ predictions.

Best estimates for the average values extracted only from the runs with the Kramers algorithm at different $\gamma$ are

$$
\begin{array}{ll}
\left\langle\Sigma_{-}\right\rangle=-0.279 \pm 0.002, & \left\langle\Sigma_{-}^{2}\right\rangle_{c}=0.189 \pm 0.001 \\
\left\langle\Sigma_{+}\right\rangle=0.316 \pm 0.002, & \left\langle\Sigma_{+}^{2}\right\rangle_{c}=0.145 \pm 0.001
\end{array}
$$

and are compatible with both the theoretical predictions and the Hybrid Monte Carlo results.

From the data obtained we see that in the negative phase $\gamma_{-}=1.0$ is the optimal value for both the operators. The same happens in the positive phase but with a smaller optimal value $\gamma_{+}=0.5$. We can see that in the negative phase the performances of the two algorithms are comparable, whereas in the positive one the Kramers algorithm is about 1.5 times slower.

The existence of an optimal range for the parameter $\gamma$ at fixed $\epsilon$ is easily understood as follows. In the $\gamma \rightarrow \infty$ the Hybrid Monte Carlo with $N_{m d}=1$ is recovered and it is well known that this is far from being an optimized regime. In the opposite limit, $\gamma \rightarrow 0$, we 
obtain the classical equations of motion in the background $\chi$ field between two refreshments. Of course molecular dynamics without noise is highly self-correlated and again a large $\tau$ results. In the Gross-Neveu model the $\gamma \rightarrow 0$ limit is particularly dangerous. We can study the $N=\infty$ model taking for $m_{c}$ the theoretical prediction including next-to-leading $1 / N$ correction on the $40^{2}$ lattice at $\lambda=2.0$. One finds that the $\Sigma$ effective potential is so unbalanced that one of the two minima disappears. In other words, if the quantum (noise) dynamics is switched off keeping all the parameters fixed, the behaviour of the system changes dramatically.

\section{SUMMARY AND CONCLUSIONS}

This work is a first numerical application of a previous investigation on the Kramers equation approach to the lattice simulations of field theoretical models. We proposed many schemes among which the simplest (introduced in [5]) is studied here. The situation which we have chosen should be one of the worst possible cases, namely a model without bosonic degrees of freedom and with a dynamical symmetry breaking mechanism which needs a peculiar determination of the critical point. The main point of our simulation is that a not so critical tuning of its parameters gives a performance comparable to that of the Hybrid Monte Carlo. This is quite important with an eye on realistic models, like $Q C D$, where a precise tuning is too expensive to be done. Moreover, the numerical precision argument makes us confident that the present algorithm can be valuable as an independent check for eventual biases of the Hybrid Monte Carlo. Simple scaling arguments (possibly naive because of the peculiar negation of momenta) indicate that the proposed algorithm should improve its performances with greater volumes or smaller fermion masses.

\section{ACKNOWLEDGMENTS}

We thank Prof. Paolo Rossi for a careful reading of the manuscript and many useful discussion. We thank Prof. Raffaele Tripiccione for a continuous help in developing the 
codes for the APE machine. We also acknowledge the support of many people from the Rome APE group, particularly Simone Cabasino and Gian Marco Todesco. 


\section{REFERENCES}

* beccaria@hpth4.difi.unipi.it, beccaria@vaxsns.sns.it

* curci@mvxpi1.difi.unipi.it

[1] M. Beccaria, G. Curci, submitted to Phys. Rev. E.

[2] S. Duane, A. D. Kennedy, B.J. Pendleton and D. Rowan, Phys. Lett. 195B, 216 (1987).

[3] M. Creutz, Phys. Rev. D38, 1228 (1988).

R. Gupta, G. W. Kilcup and S. R. Sharpe, Phys. Rev. D 38, 1278 (1988).

S. Gupta, A. Irback, F. Karsch and B. Petersson, Phys. Lett. 242B, 437 (1990).

[4] A. M. Horowitz, Phys. Lett. 156 B, 89 (1985).

A. M. Horowitz, Nucl. Phys. B280, 510 (1987).

[5] A. M. Horowitz, Phys. Lett. 268 B, 247 (1991).

[6] M. Campostrini, G. Curci and P. Rossi, Nucl. Phys. B314, 467 (1989).

[7] J. F. Schonfeld, Nucl. Phys. B95, 148 (1975).

R. G. Root, Phys. Rev. D11, 831 (1975).

[8] M. Campostrini and P. Rossi, Nucl. Phys. B329, 753 (1990).

[9] B. Rosenstein, B. J. Warr, S. H. Park, Phys. Rep. 205, 59 (1991).

[10] C. Luperini and P. Rossi, Ann. Phys. 212, 371 (1991).

[11] K. Symanzik, in Mathematical Problems in theoretical Physics, Lecture notes in physics 153, ed. R. Schrader et al. (Springer, Berlin, 1983).

[12] W. H. Press and S. A. Teukolsky, Biconjugate Gradient Method for Sparse Linear Systems in Comp. in Phys. 6, 400 (Jul/Aug 1992).

[13] M. Hestenes and E. Stiefel, Nat. Bur. of Standards Journ. Res. 49, 409 (1952). 
[14] P. Rossi, C. T. H. Davies and G. P. Lepage, Nucl. Phys. B297, 287 (1988).

[15] A. Bartoloni et al., An hardware implementation of the APE100 architecture, Int. Journ. of Mod. Phys. C, (1993) in press.

[16] A. Bartoloni et al., The software of the APE100 processor, Int. Journ. of Mod. Phys. C, (1993) in press.

[17] A. Sokal, Monte Carlo Methods in Statistical mechanics: Foundations and New Algorithms, Cours de Troisième Cycle de la Physique en Suisse Romande.

N. Madras and A.D. Sokal, Jour. of Stat. Phys. 50, 109 (1988).

[18] R. Friedberg and J. E. Cameron, J. Chem. Phys. 52, 6049 (1970) 


\section{TABLES}

TABLE I. Horowitz $\epsilon=0.09 k=6$

\begin{tabular}{lcc}
\hline \hline$\left(\Sigma_{0}, \gamma\right)$ & $\tau_{\Sigma}$ & $\tau_{\Sigma^{2}}$ \\
\hline$(-, 0.2)$ & $53(7)$ & $42(6)$ \\
$(-, 0.5)$ & $49(5)$ & $40(4)$ \\
$(-, 1.0)$ & $44(4)$ & $37(3)$ \\
$(-, 2.0)$ & $58(7)$ & $46(5)$ \\
$(-, 5.0)$ & $64(8)$ & $47(5)$ \\
$(+, 0.2)$ & $43(5)$ & $35(4)$ \\
$(+, 0.5)$ & $38(3)$ & $29(3)$ \\
$(+, 1.0)$ & $44(5)$ & $31(3)$ \\
$(+, 2.0)$ & $41(4)$ & $29(2)$ \\
$(+, 5.0)$ & $66(9)$ & $41(5)$ \\
\hline \hline
\end{tabular}

TABLE II. Hybrid (rescaled by $\mathcal{C}) \epsilon=0.06 N_{m d}=8$

\begin{tabular}{lcc}
\hline \hline$\Sigma_{0}$ & $\tau_{\Sigma}$ & $\tau_{\Sigma^{2}}$ \\
- & $44(3)$ & $32(2)$ \\
+ & $26(2)$ & $19(1)$ \\
\hline \hline
\end{tabular}

